
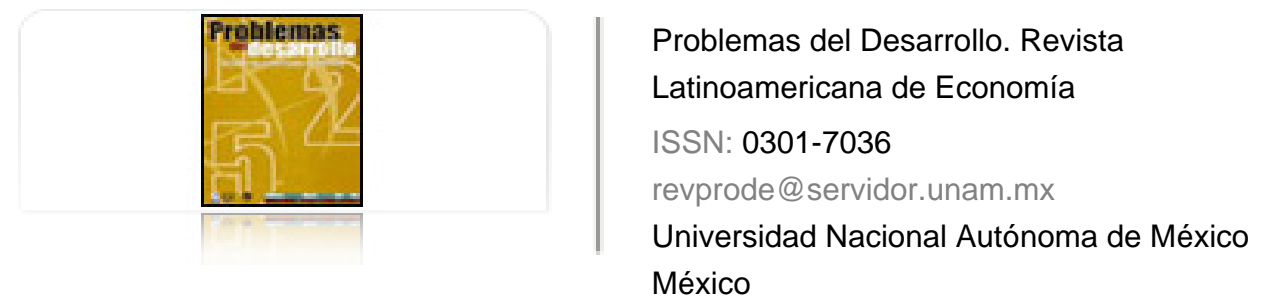

de la Reza, Germán A.

Eliminación de la medidas antidumping en el ALCA: de la "práctica desleal" al debate sobre los efectos anticompetitivos en el hemisferio occidental

Problemas del Desarrollo. Revista Latinoamericana de Economía, vol. 34, núm. 133, 2003, pp. 79-100

Universidad Nacional Autónoma de México

Distrito Federal, México

Disponible en: http://www.redalyc.org/articulo.oa?id=11825949011

Cómo citar el artículo

Número completo

- Más información del artículo

Página de la revista en redalyc.org

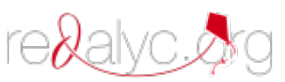

Sistema de Información Científica

Red de Revistas Científicas de América Latina, el Caribe, España y Portugal Proyecto académico sin fines de lucro, desarrollado bajo la iniciativa de acceso abierto 


\title{
ELIMINACIÓN DE LAS MEDIDAS ANTIDUMPING EN EL ALCA: DE LA "PRÁCTICA DESLEAL" AL DEBATE SOBRE LOS EFECTOS ANTICOMPETITIVOS EN EL HEMISFERIO OCCIDENTAL
}

\author{
Germán A. de la Reza*
}

Fecha de recepción: 17 de septiembre de 2003. Fecha de autorización: 27 de octubre de 2003.

\begin{abstract}
Resumen
El presente artículo examina el estado actual de la negociación de las medidas antidumping en el ALCA. Con este fin se atienden tres aspectos del problema: El debate teórico - a menudo contradictorio - de los efectos de las medidas antidumping sobre la producción y el consumo. Enseguida se examinan sus consecuencias sobre las relaciones comerciales interamericanas, poniendo de relieve la concentración de los costos en las pequeñas economías del Hemisferio. En tercer lugar, se elabora una serie de alternativas destinadas a limitar su incidencia en la profundización de las asimetrías hemisféricas. La conclusión del trabajo señala la importancia de alcanzar una definición equilibrada y atenta a las disparidades económicas y administrativas del continente.
\end{abstract}

Palabras clave: ALCA, medidas antidumping, pequeñas economías, barreras no arancelarias, libre comercio.

\begin{abstract}
This paper studies the FTAA' antidumping negotiation process. It focuses on three important sides of the problem: The theoretical background, often contradictory, regarding the antidumping effects on industry and consumers. Then, the impact of the antidumping investigations on the inter-American trade, highlighting the concentration of costs on the small economies. In third place, it draws a set of proposals voided to diminish the antidumping contribution to the aggravation of the hemispheric asymmetries. The conclusion establishes the importance of achieving a more-balanced negotiating outcome, taking into consideration the FTAA economic and administrative disparities.
\end{abstract}

Key words: FTAA, antidumping, small economies, non-tariff barriers, free trade.

Profesor investigador del Departamento de Economía de la UAM Azcapotzalco, México. Investigador Nacional. El autor agradece los comentarios y sugerencias de Efrén Marín y de tres dictaminadores anónimos. 


\section{Résumé}

Cet article examine l'état actuel de la négociation des mesures anti-dumping au sein de l'ALCA. Dans ce but, trois aspects du problème sont étudiés: tout d'abord, le débat théorique — souvent contradictoire - des effets des mesures anti-dumping sur la production et la consommation; ensuite leurs conséquences sur les relations commerciales inter-américaines, mettant en relief la concentration des coûts sur les petites économies de l'Hémisphère; en troisième point, est élaborée une série de choix destinés à limiter leur incidence sur l'approfondissement des asymétries hémisphériques. La conclusion de ces travaux signale l'importance d'atteindre une définition équilibrée et attentive des disparités économiques et de gestion du continent.

Mots-cléfs: ALCA, mesures anti-dumping, petites économies, barrières non douanières, commerce inter-américain.

\section{Resumo}

Este artigo examina o estado atual da negociação das medidas anti dumping na ALCA. Para isto, consideram-se três aspectos do problema: o debate técnico — muitas vezes contraditório- dos efeitos das medidas anti dumping sobre a produção e o consumo; imediatamente examinam-se as suas conseqüências sobre as relações comerciais inter-americanas, destacando a concentração dos custos nas pequenas economias do Hemisfério. Em terceiro lugar, elabora-se uma série de alternativas destinadas a limitar a sua incidência no aprofundamento das assimetrias hemisféricas. A conclusão do trabalho indica a importância de conseguir uma definição equilibrada e atenta das disparidades econômicas e administrativas do continente.

Palavras chave: ALCA, medidas anti dumping, pequenas economias, barreiras não tarifárias, comércio inter-americano. 


\section{Introducción}

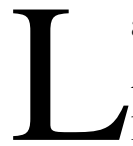

a negociación del Área de Libre Comercio de las Américas (ALCA) representa para América Latina quizá la principal oportunidad para la revisión y adecuación de las normas de comercio internacional que afectan a sus exportaciones. El proceso, iniciado en diciembre de 1994 y que en 1998 entró en la etapa formal de diseño del Tratado hemisférico con vistas a su conclusión en diciembre de 2004, alberga a la totalidad de los temas de la Organización Mundial de Comercio (OMC). A diferencia de esta última, la creación del mercado ampliado hemisférico permite el ajuste de las insuficiencias normativas multilaterales, al tiempo que el examen más preciso de las consecuencias que tienen dichas normas sobre las disparidades económicas. En ese camino, las medidas antidumping ( $\mathrm{AD})$ representan, a su vez, el aspecto central del acceso a mercados, en particular del estadounidense, y un factor de incertidumbre para el sector externo latinoamericano.

Las primeras secciones del artículo revisan los fundamentos teóricos de la operación de las $\mathrm{AD}$; las secciones centrales analizan sus significados para el comercio interamericano, sobre todo el caso de las llamadas pequeñas economías, y en final se reflexiona sobre las principales alternativas que se presentan a los negociadores estacionados en la ciudad de Puebla. Aunque la operación de las AD está conectada en última instancia con los mecanismos de solución de controversias, este artículo no los considera de manera a preservar su coherencia analítica. Tres premisas básicas guían la argumentación: el carácter fundamentalmente anticompetitivo de las $\mathrm{AD}$; la concentración de los costos directos e indirectos en las pequeñas economías, y la consecuente necesidad de una cuidadosa negociación del Capítulo sobre Subsidios, Antidumping y Derechos Compensatorios (SADC) del Borrador de Tratado del ALCA. Aunque no se desconocen los avances realizados en este sentido, en particular la inclusión del Art. 19, mencionado enseguida, se esboza una serie de alternativas procompetitivas en función de su factibilidad.

\section{Punto de partida}

Según las disposiciones del Art. vi del Acuerdo General de la OMC, adoptados por los miembros de este organismo a partir de 1994, el dumping se produce cuando el precio del producto en el país de origen (en su defecto sus costos de producción o el precio justo, estimado a partir de una canasta de precios internacionales) es mayor al precio de venta en el mercado de exportación. Si la investigación constata esta diferencia, las empresas son 
pasibles de sanciones que incluyen el pago de derechos a las firmas denunciantes, entre otros costos. ${ }^{1}$ El segundo Borrador de Tratado del ALCA, difundido el 1o. de noviembre de 2002, contiene una definición similar. Según el Art. 2 (Determinación de dumping) del SADC: ${ }^{2}$

[Se considerará que un producto es objeto de dumping, es decir, que se introduce en el mercado de otro país a un precio inferior a su valor normal, cuando su precio de exportación al exportarse de un país a otro sea menor que el precio comparable, en el curso de operaciones comerciales normales, de un producto similar destinado al consumo en el país exportador.]

Otros párrafos, divididos en 19 artículos, retoman gran parte del contenido del Acuerdo de la omc y en caso de insuficiencia normativa, sus Disposiciones generales prevén, eventualmente, la necesidad de que los países miembros defieran la investigación al organismo mundial. Aunque el Borrador todavía está sujeto a modificaciones (el elevado número de párrafos, frases y términos entre corchetes dan constancia de su carácter preliminar), puede asumirse que la mayor parte del SADC o el espíritu de sus párrafos serán definitivos. La comparación con el texto de referencia o con el Tratado de Libre Comercio de América del Norte (TLCAN) y los tratados que jalonan el Hemisferio, sustenta esta afirmación. Salvo un importante párrafo, materia de fricción entre los gobiernos y que sirve de punto de partida para el presente estudio. Según el Art. 19 (Eliminación de medidas antidumping), presente ya en el primer Borrador del 1 de julio de 2001:

[Cuando se establezca la zona de libre comercio y los bienes circulen en los países del ALCA fundamentalmente libres de restricciones, los países renunciarán al uso de medidas antidumping para el comercio recíproco.]

Quienes están familiarizados con el proceso negociador, notarán la contradicción entre el párrafo y la postura negociadora de Estados Unidos. Sus autoridades comerciales no sólo se oponen a la erradicación o a cualquier cambio en sus leyes de remedio comercial, sino que carecen de mandato negociador. Ciertamente, su presencia en el Borrador no implica que vaya a quedar en el Tratado; refleja, ante todo, el deseo de numerosos negociadores latinoamericanos de ver sustituidas las AD por políticas de competencia, una alternativa que recurre al fundamento histórico de las $\mathrm{AD}$, pero ambos bandos entienden que el disenso en este u otro tema imposibilitaría la inclusión de la normativa en el texto final. ${ }^{3}$

El objetivo de las AD es, desde una perspectiva sintética, evitar la "discriminación basada en precios desleales implementada por los productores extranjeros con el fin de obtener poder de mercado y beneficios económicos" (Russell, 1999:325). Su aplicación, prevista en las legislaciones nacionales y en todos los acuerdos de libre comercio, se inicia con la solicitud de investigación hecha por las empresas afectadas por la irrupción de im portaciones a bajo precio, a lo cual sigue la búsqueda de comprobación del dumping de las empresas denunciadas.

2 Las referencias al SADC Son tomadas de la síntesis del Anexo de este artículo. Para el texto completo véase: http://www.ftaa alca.org

3 Canadá es el primer país del mundo en adoptar una legislación antidumping en 1904. Su objetivo es protec cionista, dirigido a contener las importaciones de acero (provenientes de Estados Unidos) bajo el argumento 
Vale la pena precisar que la oposición no es propiamente entre negociadores estadounidenses y latinoamericanos; grupos empresariales y gremiales, además de gubernamentales, mantienen puntos de vista encontrados aún al interior de cada país. Desde una perspectiva analítica, el desencuentro se origina más bien en dos hipótesis opuestas sobre la naturaleza del dumping y, por extensión, de las AD.

\section{Enfoque politico del dumping}

El signo de igualdad entre dumping y práctica desleal, implícito en formulación y aplicación de las $\mathrm{AD}$, se sustenta en un conjunto de supuestos de carácter político y jurídico. ${ }^{4}$ Según este enfoque, la sinonimia está dada por las mismas condiciones que favorecen la aparición del dumping: a) posición monopólica de la empresa en el mercado nacional; b) existencia de formas de subsidio (incluyendo compras gubernamentales), y $c$ ) de una manera menos precisa, técnicas proteccionistas que permiten a la empresa ejercer control sobre los precios nacionales. De estas condiciones se derivan cuatro principales argumentos. El primero constata que la autoridad comercial de un país puede corregir las restricciones anticompetitivas en su mercado, pero carece de competencias para realizar esa labor en el ámbito internacional. El segundo advierte que si bien la venta local de un producto a precios inferiores a los costos de producción termina por agotar los recursos de la empresa, cuando esto ocurre internacionalmente le permite a la empresa beneficiarse de apoyos gubernamentales de manera indefinida (los países no quiebran). El tercero, por su parte, señala que en el mercado nacional es posible conocer en detalle las estrategias anticompetitivas, mientras que en otros mercados esto es difícil. Finalmente, que en el mercado nacional los competidores encuentran circunstancias iguales en materia de costos de capital, leyes y regulaciones sobre las inversiones, lo cual no ocurre a nivel internacional (Ragosta y Magnus, 1997). Por todo esto, se arguye que las AD son prácticamente

de la práctica desleal. W.S. Fielding, Ministro de Finanzas canadiense de la época, explicaba la normativa en términos no muy diferentes a los actuales: "El monopolio o una combinación de empresas, habiendo obtenido el comando y el control de su propio mercado, y encontrando que tiene una sobreoferta de productos, busca obtener el comando del mercado vecino y tiene como objetivo su control [...] Estos [productores] envían los productos [al Canadá] con la esperanza y la expectativa de arrojar [del mercado] a las industrias nativas cana dienses" (citado en Grey, 1986:11 12). El segundo país es Estados Unidos, cuya Acta Antidumping de 1916 representa una extensión de leyes antimonopólicas anteriores; recién en 1921 incorpora el concepto de "daño producido por las importaciones". El origen distinto de las AD es una ilustración, según Finger (1993:13), de lo que son las leyes de remedio comercio (simple proteccionismo) y de lo que no pueden ser (una extensión de las políticas antimonopólicas). Hacia 1921, sólo cuatro países más disponían de esta normativa (Australia, Nueva Zelanda, Gran Bretaña y Francia); habrá que esperar hasta la Ronda Kennedy (1965 1967) para que se convierta en un tema importante. Para un número de países en desarrollo, la incorporación de legislaciones específicas empieza entonces; otros las adoptan luego de la conclusión de la Ronda Uruguay en 1994.

$4 \quad$ La argumentación favorable a las medidas antidumping es numerosa. Véase, entre otros: Garten (1994); Howell (1998) y Mastel (1998). En estos trabajos el concepto de dumping se utiliza a menudo bajo la connotación estratégica, respuesta a las dificultades que encontraron los defensores de las AD en los años de 1980 para lograr el apoyo del Congreso estadounidense. Su principal característica es el establecimiento de un vínculo entre el dumping y la idea de "mercados extranjeros cerrados a la competencia internacional". 
indisociables del comercio internacional en la tarea de corregir el comportamiento dañino de algunos exportadores.

Previo a la consideración del enfoque económico, revisemos brevemente estos supuestos y sus implicaciones. El primer argumento es compartido por un amplio número de analistas, para quienes el reemplazo por políticas de competencia no es un objetivo inmediato, sino que interviene luego de la liberalización arancelaria, cuando es posible que operen políticas de competencia homogéneas. El segundo se fundamenta en las limitaciones de los acuerdos de libre comercio en materia de compras gubernamentales y de apoyos directos e indirectos a las empresas menos competitivas. Al igual que la primera, esta objeción es compartida por analistas serios y refuerza la necesidad de adoptar las regulaciones del caso. La tercera y cuarta premisa, por el contrario, suponen que la heterogeneidad o el desconocimiento de los mercados implican per se la legitimidad de las AD. El desconocimiento es difícil de aceptar como argumento válido; asume la general preferencia de las empresas por el dumping y la amplitud de su cobertura implica situaciones que poco o nada tienen que ver con esta estrategia. Igual reparo plantea hacer responsable a las empresas de las características de su mercado nacional. Por el contrario, la estrechez de los mercados de capitales y la proliferación de regulaciones sobre la actividad empresarial (entre decretos, certificados y otros trámites) afectan sobre todo a países en desarrollo que hacen un escaso uso de las AD. ${ }^{5}$ Aquí es necesario precisar que la principal objeción que puede hacerse al enfoque político no se refiere a la interpretación del dumping, sino al hecho de ignorar el impacto anticompetitivo de las AD.

\section{El enfoque económico}

La hipótesis económica del dumping se origina en el estudio seminal de Viner (1923) sobre las implicaciones de la Acta Antidumping estadounidense aprobada en $1921 .^{6}$ Este trabajo establece la definición durable del dumping como la discriminación internacional de precios esporádica, intermitente o permanente que adoptan las empresas por motivos diversos, en un abanico que va desde estrategias puramente comerciales, hasta la búsqueda de eliminar a la producción rival. Para le explicación de la hipótesis económica utilicemos un modelo de discriminación de precios en condiciones de competencia imperfecta y mercados segmentados (cuando el consumidor nacional no puede acceder a los bienes

\footnotetext{
Un reciente estudio del Banco Mundial (2003) observa la relación entre el número de trámites, decretos y regulaciones que debe realizar una empresa para su registro, y el menor desarrollo de los países. en Bolivia son necesario 18 trámites y 67 días; 14 trámites y 90 días en Ecuador, 12 trámites y 115 días en El Salvador. Otros países con elevado número de regulaciones son: Brasil, México, Argentina, Haití, Honduras, Paraguay. En Estados Unidos son necesarios sólo 5 trámites y 4 días.

6 Viner no sólo pone las bases del estudio económico del dumping, sino que es uno de los primeros en señalar las consecuencias anticompetitivas de las AD. En su discurso de 1955 ante el Congreso estadounidense, advierte que las empresas "pueden, si así lo desean, elevar la las barreras arancelarias efectivas más de lo que las negociaciones [del GATT] puedan lograr en otra dirección".
} 
dedicados a la exportación). Sea, pues, una empresa monopolista $(E)$, un mercado nacional $(M n)$ y otro de exportación $(M e)$. Asumimos que las exportaciones de $E$ son altamente sensibles al precio externo ( $P e)$ y que $M n$ y $M e$ están suficientemente segmentados como para permitir la situación de dumping: $P n>P e$. Siguiendo la lógica de maximización de beneficios, $E$ buscará igualar el ingreso marginal en el $M n$ al coste marginal de ambos mercados. Con este fin producirá una cantidad de bienes suficiente para satisfacer la demanda nacional y exportar el resto. Obsérvese que la fabricación de todo producto adicional es igual a $P e$, constante en razón de la elasticidad en la demanda de $M e$ (siempre mayor a la elasticidad de $M n$ ). Asimismo, que el aumento de las ventas en $M n$ tiende a reducir el $P n$, eliminando el estímulo a la comercialización nacional. En estas condiciones, la diferencia $P n-P e$ ocurre en función del ingreso marginal y en cantidades de producción determinados por éste. Su valor frecuentemente toma en consideración el tipo de consumidor (rico o pobre), del lugar de abastecimiento (cercano o alejado) y del tipo de consumo (mayorista o minorista).

En otros términos, la discriminación de precios representa una distorsión dañina para la industria sólo en el caso del dumping predador (corto plazo), aunque el conjunto de los casos de discriminación favorece la creación de comercio. En ese sentido, puede decirse que representa una estrategia legítima de comercio internacional. A esto se agrega que el establecimiento de un precio uniforme $(P n-P e=0)$ no está exento de distorsiones. Cuando se obliga a las empresas a cargar un precio uniforme, uno de los fenómenos que podría generar sería el "quitar a $X$ para dar a $Y$ ". Mas generalmente, la aplicación del precio uniforme conduciría a que uno de los mercados no esté servido dado que el precio sería igual a $P n$, menos competitivo en el caso que analizamos. Algunos aspectos reales del comercio apoyan nuestras conclusiones: si el exportador enfrenta un contexto internacional de sobreoferta, es posible que reduzca sus precios (hasta donde lo permita el ingreso marginal) sin por ello buscar una estrategia de dumping. Igual necesidad puede plantear un escenario de recesión económica o de incremento en la competitividad internacional, sobre todo si el exportador decide mantenerse en el mercado. En otro orden de ideas, el actual predominio del comercio intraindustrial implica la posibilidad del dumping recíproco; una especie de suma cero en los beneficios que puede reportar el dumping. ${ }^{7}$ Por último, si la principal condición para la existencia de dumping es la operación de monopolios, el problema debe atenderse a través de políticas de competencia, más eficientes y especializadas en el conjunto de sus distorsiones, y no mediante una investigación que en pocas ocasiones conduce a evidencias determinantes.

Sobre el argumento del dumping recíproco y sus consecuencias sobre el bienestar, véase Brander (1981). 


\section{El nuevo proteccionismo}

La ausencia de efectos particularmente negativos en el dumping desplaza la atención hacia el otro componente del problema: el efecto de las AD. No cabe duda que éste es principalmente proteccionista, estimulado de manera significativa por haber sustituido a los aranceles como instrumento de control del comercio internacional. Mientras el promedio arancelario mundial pasó de 50 en 1958 a sólo 5\% en el 2000, las investigaciones AD crecieron desde los años ochenta en una proporción doble a la década precedente. En estas condiciones y a pesar de que las AD tienen una naturaleza específica, su utilización proteccionista no está alejada del problema de anticompetitividad que plantean los aranceles: perjuicio al bienestar y fomento a la ineficiencia. Casi cada estudio cuantitativo ha puesto de relieve su función como proteccionismo furtivo, divorciado frente a las consideraciones de bienestar nacional. ${ }^{8}$ Más recientemente, la existencia de amplias series estadísticas ha permitido observar que las AD no eliminan globalmente el dumping, sino que el efecto dominó impulsado por el alejamiento de la dinámica de libre comercio favorece la promiscuidad de su empleo. ${ }^{9}$

El principal problema de las AD para los productores y los gobiernos son los costos y la variedad de fuentes donde éstos se originan. Una de estas se localiza en el periodo de revisión, cuando las empresas alteran su comportamiento para influir en el resultado de la investigación. Otra fuente son las Restricciones Voluntarias a las Exportaciones (RVE). La lógica de las RVE como barrera no arancelaria se refiere al caso cuando un exportador reduce voluntariamente sus ventas como consecuencia de la amenaza de nuevas restricciones comerciales en el mercado de exportación. Según los acuerdos de la Ronda Uruguay, estaba prevista su eliminación para el año 2004 (diez años de transición), pero no existen señales de que pronto vaya a abandonar la zona gris de violaciones al acuerdo posibilitadas por su insuficiencia normativa. De manera más visible, la tercera categoría de costos sobreviene cuando la empresa investigada paga los derechos que dicta la autoridad comercial del país importador. Son de sobra conocidos en el ámbito empresarial y se han convertido en una amenaza permanente para los exportadores. Incluso en el Congreso estadounidense - instancia que aglutina a importantes críticos del dumping — es un secreto a voces que las AD son el "arma preferida del proteccionista" (Dam, 2001:148). Provistos de estas consideraciones, desglosemos el impacto de las AD en el hemisferio occidental.

\footnotetext{
El párrafo corresponde a las conclusiones de un influyente estudio de Stiglitz (1997). Dam (2001) desarrolla una argumentación muy cercana.

9 Esta relación ha sido observada por Anderson (1992). Para la confirmación de la hipótesis del efecto dominó, véase Blonigen y Park (2000).
} 
Cuadro 1

Indicadores de las AD estadounidenses

\begin{tabular}{lcc}
\hline & $1990-1994$ & $1995-1999$ \\
\hline AD iniciadas (promedio anual) & 53 & 26 \\
AD sancionadas (promedio anual) & 23 & 16 \\
AD en activo (al final del periodo) & 326 & 267 \\
De EE.UU./Contra EE.UU. & $281 / 79$ & $267 / 107$ \\
Derechos sancionados (\%) & 56.2 & 46.7 \\
Duración (promedio) & 7 años & 8 años \\
\hline
\end{tabular}

Fuente: сво (2001) у USTC (2003).

\section{Costos en el Hemisferio Occidental}

Entre 1987 y 2000, Estados Unidos recurrió en 147 ocasiones a las AD contra importaciones provenientes de América Latina y el Caribe, casi una investigación nueva por mes. Sólo en el año 1990, éstas tuvieron un costo —en equivalentes arancelarios- a 25\% de las ventas de textiles, metales ferrosos, acero y televisión a color; $30 \%$ en el caso del arroz y $40 \%$ de las exportaciones de azúcar. ${ }^{10}$ En el caso de las AD aplicadas a las ventas canadienses, aunque afectaron una pequeña fracción del comercio bilateral $(0.16 \%$ de las importaciones estadounidenses), el promedio de los derechos fue de 25\% (UsITC, 1995). El examen del resto de la década sugiere que la aplicación de esta normativa llegara a tener un efecto incluso mayor a los aranceles. Cuando el dumping fue demostrado (en $46.7 \%$ de los casos iniciados entre 1995 y principios del 2000), los costos variaron entre 50 y $70 \%$; cuando la investigación no lo reveló (53.3\% de los casos en el mismo periodo), la pérdida fluctuó entre 15 y 20\%. ${ }^{11}$ En octubre de 2003 estaban vigentes 43 investigaciones AD contra productos hemisféricos, en principal los derivados de la industria metalúrgica, bienes agrícolas, prácticos y cemento en proveniencia de Argentina, Brasil, Canadá, México, Trinidad y Tobago, y Venezuela. ${ }^{12}$

A esto hay que agregar el factor de incertidumbre en el acceso a mercados. Debido a la relativa facilidad con la que las empresas pueden solicitar en Estados Unidos una investigación antidumping y al propio hecho de su duración (267 activas a principios del 2000, con una duración promedio de 7 a 8 años), las extranjeras se han visto a menudo en la necesidad de restringir sus exportaciones para evitar el desgaste administrativo que acarrea el proceso legal (véase Cuadro 1). Estas concesiones (o RVE) están ampliamente presentes en la suspensión de los casos estadounidenses y en cierta medida pueden considerar-

10 Sobre la equivalencia arancelaria y su metodología, véase Erzan y Yeats (1992:59ss).

11 El desglose de los costos cuando no se prueba el dumping se encuentra en Prusa (1999). Estos son mencionados en el presente artículo: modificación del comportamiento comercial y desgaste administrativo.

12 Para una relación actualizada de las AD vigentes, véase usitc, http//: www.usitc.gov.org 
se como un componente estable de la estrategia de sus empresas. Entre 1980 y 1994, de 20 a $25 \%$ del total de las investigaciones originadas en ese país concluyeron en RVE sin que hubiera serios indicios de dumping. Esta situación se mantiene hasta nuestros días a pesar de las mejoras en materia de determinación de dumping contenidas en los acuerdos de la Ronda Uruguay. ${ }^{13}$

\section{El reverso del proteccionismo}

Las investigaciones antidumping también tienen efectos costosos en el país donde se inicia el caso. El estudio más completo sobre su impacto en la economía estadounidense, realizado por la Comisión de Comercio Internacional (ITC, por sus siglas inglesas) en 1995, muestra que la eliminación de las AD que estaban activas en 1991, hubiera incrementado en ese año el bienestar estadounidense en 6 mil millones de dólares (ITC, 1995). Otro estudio, realizado en el marco de la Comisión Federal de Comercio por Morkre y Kelley (1994), sostienen esta conclusión respecto al periodo 1980-1988. Uno más reciente, preparado en la División de Gobierno y Finanzas y presentado al Congreso estadounidense (26 de junio del 2002), insiste en el perjuicio económico que ocasionan las AD a los consumidores estadounidenses, así como en el hecho que su aplicación no inhibe el dumping (Elwell 2002). Este cúmulo de evidencias señala con claridad que las leyes de remedio comercial representan "cargas substanciales [para los] consumidores y [los] exportadores [estadounidenses] más eficientes, al tiempo que protegen a [las] empresas menos competitivas frente a la exportación" (Stiglitz, 1997:418). ${ }^{14}$

Un costo adicional para Estados Unidos, relativamente nuevo, resulta de su (¿involuntaria?) contribución al desarrollo de este obstáculo comercial. Entre 1990 y 2000 se iniciaron en el mundo 2,483 investigaciones AD, poco más del doble que en la década precedente, con la particularidad que a partir de 1995 se incrementaron las investigaciones contra de firmas estadounidenses. La situación no es distinta en el hemisferio occidental; entre 1987 y 2000 Estados Unidos inició 147 investigaciones, mientras que en ese mismo lapso sus empresas fueron objeto de 182 medidas AD. Obsérvese que este cambio de tendencia influye cada vez más en el diseño de las estrategias gerenciales de ese país y que presenta un desafío para el conjunto del sector externo. ${ }^{15}$ No obstante, la representación de

13 Los avances operados por la Ronda Uruguay incluyen nociones más precisas para la determinación del dumping y para probar el daño comercial. Otro aspecto importante es el refuerzo del mecanismo de solución de controversias: en la actualidad las resoluciones de la omc tienen carácter vinculante. Para el estudio sobre las modificaciones de la OMC, véase Hoeckman y Mavrodis (1999).

14 Otro país que recurre frecuentemente a las leyes de remedio comercial, Canadá, también observa efectos adversos sobre la competitividad de las importaciones y el bienestar general. Dutz (1998) señala como muy probable que el costo para los consumidores sea más elevado que los montos establecidos en el cálculo directo, en razón de su concentración en la industria de productos primarios.

15 Lindsey e Ikenson (2001) construyen una argumentación similar sobre las consecuencias de las investigaciones AD contra los productores estadounidenses. 
ese país en las negociaciones del ALCA no ha retenido las evidencias sobre la compleja y elevada anticompetitividad de las $\mathrm{AD}$, sino su costo relativamente pequeño para este país (6 mil millones de dólares equivalen a $0.03 \%$ de su PIB), en contraste con el substancial apoyo político del que benefician en el Congreso de este país.

\section{Correlaciones con la disparidad económica}

Un aspecto menos atendido por la literatura de especialidad concierne a las disparidades administrativas. De todas las investigaciones antidumping iniciadas en América Latina entre 1987 y 2000, 103 correspondieron a México, 61 a la Argentina y 40 al Brasil. Esto confirma la democratización de esta normativa y su empleo cada vez más activo en países antaño ajenos a su dinámica. Sin embargo, el paso de la protección arancelaria al uso intensivo de las regulaciones contra el comercio desleal no es el hecho de la inmensa mayoría de países del hemisferio. Veinte países de América Latina y el Caribe todavía no han iniciado ninguna investigación antidumping, y otros ocho las han emprendido en siete o menos ocasiones, una cada dos años en el mejor de los casos; a su vez, 17 países de América Latina y el Caribe no han sido objeto de investigación AD y otros 9 lo han sido en 4 o menos ocasiones (véase Cuadro 2). He ahí un desafío importante para el ALCA y un punto de reflexión para el diseño del SACD, en la medida que la liberalización arancelaria propuesta por este esquema y el consecuente aumento del comercio no dejarán de repercutir en la proliferación de las AD en el hemisferio.

Aunque el conjunto de la región ha adoptado los acuerdos de la OMC sobre prácticas desleales, en numerosos casos su aplicación muestra lagunas operativas, escasez de asistencia jurídica y limitaciones administrativas que incluyen la ausencia de manuales de procedimiento. Para estos países, la promoción del comercio hemisférico los encuentra en una situación desventajosa. Un aspecto significativo de esta dificultad es su correlación con el tamaño de las economías. Del conjunto de economías medias y pequeñas del hemisferio, sólo cinco (Colombia, Costa Rica, Perú y Venezuela) tienen alguna experiencia en la compleja normatividad de las AD; el resto, compuesto de países centroamericanos, caribeños y algunos de Sudamérica — precisamente los más pequeños— carecen de ella. ${ }^{16}$ En el otro extremo, los miembros del Tratado de Libre Comercio de América del Norte (TLCAN) acumulan, junto con Argentina y Brasil, 90\% del total de denuncias en el hemisferio. Esto deja a dichas naciones como los únicos conocedores de una ley comercial que, si no sufre reformas, podría ser clave para la estabilidad en el acceso a mercados. Un aspecto de esta correlación permite profundizar en las dificultades de las pequeñas economías: dado que

16 La definición del término pequeñas economías carece de rigor en el ALCA y a menudo se aclara que constituye una decisión política. No obstante, con indiferencia de los criterios puede asumirse que la totalidad de los países centroamericanos, caribeños y algunos de América del Sur (Bolivia, Ecuador, Paraguay y Uruguay) entran en esta categoría. 
Cuadro 2

Casos de antidumping en el hemisferio occidental (1987-2000)

\begin{tabular}{|c|c|c|c|c|}
\hline País origen & Demandante & Porcentaje & Investigado & Porcentaje \\
\hline Antigua y Barbudas & - & - & - & - \\
\hline Antillas Holandesas & - & - & - & - \\
\hline Argentina & 61 & 12.6 & 22 & 4.5 \\
\hline Aruba & - & - & - & - \\
\hline Bahamas & - & - & - & - \\
\hline Barbados & - & - & - & - \\
\hline Belice & - & - & - & - \\
\hline Bolivia & - & - & 1 & 0.2 \\
\hline Brasil & 40 & 8.3 & 104 & 21.5 \\
\hline Canadá & 84 & 17.3 & 48 & 9.9 \\
\hline Chile & 5 & 1.0 & 16 & 3.3 \\
\hline Colombia & 11 & 2.3 & 11 & 2.3 \\
\hline Costa Rica & 6 & 1.2 & 2 & 0.4 \\
\hline Dominica & - & - & - & - \\
\hline Estados Unidos & 147 & 30.4 & 182 & 37.6 \\
\hline Ecuador & 1 & 0.2 & 4 & 0.8 \\
\hline El Salvador & - & - & - & - \\
\hline Granada & - & - & - & - \\
\hline Guatemala & 1 & 0.2 & 1 & 0.2 \\
\hline Guyana & - & - & - & - \\
\hline Haití & - & - & - & - \\
\hline Honduras & - & - & 1 & 0.2 \\
\hline Jamaica & - & - & - & - \\
\hline México & 103 & 21.3 & 54 & 11.2 \\
\hline Nicaragua & 2 & 0.4 & 1 & 0.2 \\
\hline Panamá & 1 & - & - & - \\
\hline Paraguay & - & - & 2 & 0.4 \\
\hline Perú & 11 & 2.3 & 2 & 0.4 \\
\hline República Dominicana & - & - & - & - \\
\hline Saint Kitts & - & - & - & - \\
\hline Santa Lucía & - & - & - & - \\
\hline San Vicente & - & - & - & - \\
\hline Trinidad y Tobago & 4 & 0.8 & 3 & 0.6 \\
\hline Uruguay & - & - & 3 & 0.6 \\
\hline Venezuela & 7 & 1.4 & 28 & 5.8 \\
\hline
\end{tabular}

los costos del proceso de revisión son fundamentalmente absorbidos por los gobiernos, es necesario que el país disponga de cierta capacidad financiera y administrativa. En otros términos, la habilidad para hacer frente a los costos del comercio administrado depende de tamaño económico. Y el gasto que entraña la resolución de los litigios comerciales es comparativamente más elevado en las pequeñas economías. ${ }^{17}$

${ }_{17} \quad$ Un principal estudio en la materia, conducido por el Grupo Independiente de Expertos en Pequeñas Economías e Integración del Hemisferio Occidental, muestra que la menor habilidad de las economías pequeñas respecto al comercio administrado puede explicarse, al menos en parte, por el mayor costo per cápita que tiene la resolución de los litigios comerciales (IGE, 1997). 


\section{Alternativas en la mesa de negociaciones}

En resumen, la utilización de las AD como un instrumento de control del comercio entraña riesgos importantes para el conjunto de las economías del Hemisferio, en particular para las de América Latina y el Caribe. Para estos últimos la reforma de las leyes de remedio comercial es un objetivo clave para el acceso estable al mercado estadounidense, así como para reducir el estrés administrativo y, en general, su vulnerabilidad comercial. Estas circunstancias han sido observadas por varios gobiernos de América Latina y su preocupación ha quedado parcialmente asentada en el Art. 15 del SADC:

[Se reconoce que los países desarrollados Parte del Acuerdo del ALCA deberán tener particularmente en cuenta la especial situación de los países en desarrollo Parte del Acuerdo del ALCA cuando contemplen la aplicación de medidas antidumping [o compensatorias] en virtud del presente Acuerdo. Antes de la aplicación de derechos antidumping [o compensatorios] se [hará a los exportadores una propuesta de compromiso de precios o se dará oportunidad para que el país exportador elimine los programas de subvención, según sea el caso. La autoridad investigadora dará oportunidad adecuada para celebrar consultas en las que se discuta la propuesta de compromiso de precios o eliminación de los programas de subvención o cualquier otra solución constructiva permitida por este Acuerdo u otros tratados internacionales.]

Nótese que esta normativa elimina si acaso el factor sorpresa. Deja casi intacto el problema de los costos para las economías de la región y sus estipulaciones adolecen del mismo problema de operatividad que tiene el texto de referencia, un párrafo casi idéntico del Acuerdo General de la omc. Este último, objeto de reclamos en varias ocasiones (una de ellas en febrero de 2003 ante el Comité de Negociaciones Comerciales), mostró que sus imprecisiones permiten a las autoridades comerciales un amplio espacio de interpretación. $^{18}$

Para avanzar en una solución integral, los negociadores latinoamericanos han propuesto una serie de modificaciones resumidas en el mencionado Art. 19 sobre Eliminación de medidas antidumping. Su planteamiento esgrime el argumento tradicional de la teoría económica: dado que las AD constituyen una implícita regulación proteccionista, su función debe ser reemplazada por políticas de competencia. ${ }^{19}$ Cabe mencionar que esta alternativa cuenta con dos antecedentes de importancia, la Unión Europea y la zona de libre comercio Australia-Nueva Zelanda. En éstas, el reemplazo tuvo lugar una vez concluido el proceso de liberalización comercial, y fue concomitante con la homologación de las

18 El Art. 15 del Acuerdo General de la omc estipula: "Se reconoce que los países desarrollados Miembros deberán tener particularmente en cuenta la especial situación de los países en desarrollo Miembros cuando contemplen la aplicación de medidas antidumping en virtud del presente Acuerdo. Antes de la aplicación de derechos antidumping se explorarán las posibilidades de hacer uso de las soluciones constructivas previstas por este Acuerdo cuando dichos derechos puedan afectar a los intereses fundamentales de los países en desarrollo Miembros".

19 Tavares et al. (2001) proponen una alternativa distinta: la sustitución de las AD por salvaguardas en atención a su función de alivio frente a las importaciones. 
políticas de competencia de los países miembros. Su respaldo en América Latina es inequívoco. En la reunión del Comité de Negociaciones Comerciales del ALCA en Lima en enero del 2001, los países del MERCOSUR advirtieron que no aceptarían los resultados de la negociación si las medidas antidumping no formaban parte de la agenda. En adelante, negociadores y analistas de la región han insistido en numerosas ocasiones, incluyendo la Reunión Ministerial de Cancún de la OMC en 2003, sobre la importancia de reformar las leyes de remedio comercial.

\section{Otras alternativas}

Sin embargo, es necesario destacar la existencia de varios obstáculos que no favorecen el escenario propuesto por el Art. 19 del Borrador. El primero es el referido apoyo político del que benefician las AD en Estados Unidos, el socio del cual se esperan las mayores concesiones en la materia. De manera conexa, la existencia de un candado negociador. La Autoridad de Promoción Comercial (TPA, por sus siglas inglesas) fue aprobada en 2001 con un recorte destinado a impedirle al Ejecutivo la posibilidad de negociar las leyes de remedio comercial, limitación apoyada no sólo por el sector patronal agrícola y metalúrgico, sino por las mayores organizaciones sindicales del país, la Federación Laboral Americanael Congreso de Organizaciones Industriales (AFL-CIO) y la Unión de Trabajadores Metalúrgicos de América (USWA), entre otros. ${ }^{20}$ No sorprende, en este contexto, que los representantes estadounidenses se hayan declarado en la mesa de negociaciones, una y otra vez, vigorosamente opuestos a cualquier cambio o enmendadura de las leyes de remedio comercial. Más aún, han hecho de su conservación una meta en el ALCA bajo el argumento de que las AD y las políticas de competencia se refieren a problemas distintos y, por consiguiente, no son intercambiables (USTR, 2001).

La segunda tiene que ver con las dificultades de implementar un cambio de la magnitud propuesta por el Art. 19. ${ }^{21}$ En las dos regiones donde se han sustituido las AD por políticas de competencia, ha sido necesario un proceso de dilatados tiempos. De acuerdo al Borrador de Tratado, la eliminación de la normativa tendría que esperar al menos hasta el lustro 2015-2020, cuando la totalidad del comercio (incluidas las industrias sensibles) esté libre de aranceles. Sin embargo, la actual redacción del artículo deja abierta la posibilidad de que se invoque indefinidamente la presencia de otras restricciones. Su lectura a

20 Según estos grupos gremiales, el ALCA debe implementar "leyes antidumping y derechos compensatorios nacionales que sean efectivos y transparentes para garantizar que el comercio internacional sea justo y equilibrado". Critican el Borrador del Tratado incluso cuando retoma el contenido del Art. vi del Acuerdo General de la омc, en la medida que este último redujo "la capacidad del gobierno de implementar estas leyes". El objetivo proteccionista se sugiere más adelante, cuando menciona que el acuerdo hemisférico "no debe infringir, en modo alguno, los derechos de los países a proteger sus industrias, sus trabajadores y agricultores frente a las prácticas comerciales desleales" (AfL cio y uswA, 2003).

21 Sobre las dificultades prácticas del reemplazo de las AD por políticas de competencia en Norteamérica, así como la importancia de adoptar un enfoque por etapas, véase: Leycegui y Vega Cánovas (1997: 281 y s.). 
la luz de los argumentos favorables a las AD (reseñados al inicio de este estudio), permitirían su inclusión en la zona gris de violaciones indirectas al acuerdo en virtud de sus notables imprecisiones. En más de un sentido el concepto fundamentalmente libre de restricciones resulta difuso y puede entenderse como una situación de (imposible) competencia perfecta. Si el párrafo se aprueba sin especificaciones concretas o, también probable, no es aceptado en el ALA, las AD extenderán su vigencia más allá del periodo de transición del ALCA y no dejarán de constituir una fuente de estrés administrativo e incertidumbre para la mayoría de los países de América Latina. ${ }^{22}$

Algunas alternativas procompetitivas que pueden considerarse son:

1) Incluir en el Art. 19 la referencia a un Comité de evaluación del grado de liberalización necesario para proceder a la eliminación de las AD. El TLCAN incluyó una instancia similar, aunque el carácter de recomendación que tienen sus resultados le ha restado significado. En ese sentido, es importante especificar que el dictamen del Comité tenga carácter vinculante.

2) Incrementar los criterios de existencia de daño. Aunque importa el no permitir ni considerar un examen que se fundamente en la utilización de estadísticas sobre grupos agregados, es necesaria una definición positiva del tipo de información aceptable. Para reforzar este párrafo debe acompañarse de mayor rigor en la determinación de la existencia de dumping, realizada parcialmente en la actual versión del SADC.

3) Elevar los requerimientos para la operación del concepto de rama de producción nacional. De esa manera se limitaría la notoria facilidad con la que se levantan las denuncias.

4) Introducir el concepto de costos implícitos sobre una cantidad acordada por los miembros en función de los gastos y pérdidas de las empresas durante el periodo de investigación. Este monto se restaría de los derechos que dicte la autoridad comercial o, en caso de acuerdo entre las empresas, de los compromisos del exportador.

5) Precisar el Art. 15 respecto del compromiso de precios que se propondrá a los países en desarrollo. La finalidad es evitar que este compromiso surja de las reuniones de consulta de manera similar a como se establecen las RVE. Aquí sería necesario invocar los compromisos de la omc relativos a la eliminación de esta barrera.

\section{Conclusiones}

A pesar de que no están despejadas todas las incógnitas sobre la creación del ALCA, la negociación a nivel hemisférico de las principales normas de comercio internacional ofre-

22 Según Horlick y Shea (1997:276), la eliminación de las AD requiere de instituciones supranacionales capaces de administrar esa normativa hacia terceros países. Después de reflexión nos parece que sea un obstáculo menor; el ALCA no se propone convertirse algún día en una unión aduanera con institucionalidad supranacional; para su modelo basta con adoptar políticas de competencia en combinación con el uso independiente de las AD respecto a terceros países. Un ejemplo representa la Closer Economic Relationship (CER) Australia Nueva Zelanda. 
ce a América Latina la oportunidad de corregir algunas de sus insuficiencias y distorsiones. Una de las más importantes, por sus efectos directos e indirectos, son las AD, caracterizadas por un nivel de complicación técnica que exige a su vez una negociación cuidadosa y creativa. La propuesta latinoamericana de reemplazar las AD por políticas de competencia se beneficia de un sólido sustento teórico y empírico, aunque su transposición en el Borrador de Tratado señala el riesgo de no alcanzar resultados tangibles en la materia. Aunque este artículo comparte el espíritu del Art. 19 del Borrador, expone la necesidad de precisar su contenido y operatividad, así como de elaborar alternativas procompetitivas susceptibles de contrarrestar la probable ineficacia de su redacción. La importancia que ha cobrado esta normativa como barrera no arancelaria recomienda la combinación de escenarios que aseguren la disminución de los efectos proteccionistas de las AD. La atención a las negociaciones del SADC se recomienda por otra razón importante: constituye uno de los pocos espacios en los cuales Estados Unidos puede hacer concesiones importantes, ya que el proceso de liberalización arancelaria constituye globalmente una concesión latinoamericana hacia este país. ${ }^{23}(9$

\section{Bibliografía}

AFL-CIO y uswa, Comentarios de la Federación Estadounidense del Trabajo-Congreso de Organizaciones Industriales y de United Steelworkers of America, Comité de Representantes Gubernamentales sobre la Participación de la Sociedad Civil del ALCA, CIV-94, OEA, 1o. de mayo, 2003.

Anderson, J.E., "Domino Dumping, I: Competitive Exporters", American Economic Review, vol. 8, núm. 1, 1992, pp. 65-83.

Bhagwati, J., Protectionism, Cambridge, MIT Press, 1988.

Blonigen B.A. y J.-H. Park, "Dynamic Pricing in the Presence of Antidumping Policy: Theory and Evidence", Working Paper, Oregon, Department of Economics, University of Oregon, 2000.

Blonigen, B.A. y T.J. Prusa, "The Cost of Antidumping: The Devil is in the Details", World Economics (en prensa), 2003.

Brander, J., "Intraindustry Trade in Identical Commodities", Journal of International Economies, núm. 11, 1981, pp. 1-14.
CEPAL, Barriers to Latin American and Caribbean Exports in the U.S. Market (1998-1999), (LC/WAS/ L. 55), Washington D.C., noviembre 23, 1999.

Dam, K. W., The Rules of the Global Game. A New Look at US International Economic Policymaking, Chicago, The University of Chicago Press, 2001.

De la Reza, G.A., "El ALCA y la cooperación hemisférica”, Revista de Comercio Exterior, Bancomext, agosto, México, 2003.

, Liberalización del comercio en el hemisferio occidental, México, Instituto de Investigaciones Económicas, UNAM, 1998.

Dutz, M., "Economic Impact of Canadian Antidumping", R.Z. Lawrence (editor), Brookings Trade Forum 1998, Washington, D.C., Brookings Institution Press, 1998.

Elwell, C.K., "Dumping of Exports and Antidumping Duties: Implications for the U.S. Economy", Report for Congress RL 31468, Washington D.C., Congressional Research Service, 26 de junio, 2002.

23 El problema no radica exclusivamente en el esfuerzo de adaptación a una nueva estructura de precios, sino en la elevada dependencia fiscal de estos países frente a sus ingresos arancelarios. Véase De la Reza (2003). 
Erzan R. y A. Yeats, "Free Trade Agreements with the United States -What's in it for Latin America?", Policy Research Working Paper Series 827, Banco Mundial, enero, 1992.

Finger, M.J., Antidumping: How It Works and Who Gets Hurt, Ann Arbor, The University of Michigan Press, 1993.

Garten, J.E., "New Challenges in the World Economy: The Antidumping Law and U.S. Trade Policy", Remarks made before the U.S. Chamber of Commerce, 7 de abril, Washington, D.C., 1994.

Grey, R.C., "Trade Policy and the System of Contingency Protection in the Perspective of Competition Policy", Department of External Affairs, Otawa, mayo, 1986.

Hoeckman, B. y P. Mavrodis, "wTo Dipute Settlement, Transparency and Surveillance", World Bank, 19 de noviembre, 1999.

Horlik, G.N. y E.C. Shea, "Alternativas a las leyes nacionales de Antidumpingy de cuotas compensatorias: Su viabilidad o su conveniencia en el contexto del TLCAN", B. Leycegui, W.B.P. Robson y S.D. Stein (editores), Comercio a golpes. Las prácticas desleales de comercio internacional bajo el TLCAN, México, ITAM-Miguel Angel Porrúa, 1997.

Howell, T.R., "Trade Remedies: A U.S. Perspective", Trade Strategies for a New Era: Ensuring U.S. Leadership in a Global Economy, Nueva York, Council on Foreign Relations, 1998.

Hufbauer y K.A. Elliott, Measuring the Costs of Protec-tion in the United States, Washington D.C., Institute for International Economics, 1994.

IGE, Overcoming Obstacles and Maximizing Opportunities, Independent Group of Experts on Smaller Economies and Western Hemispheric Integration, University of the West Indies, Mona, 1997.

Leycegui, B. y G. Vega-Cánovas, "Eliminating 'Unfairness' within the North American Region: A Look at Antidumping", M. Hart (editor), Finding Middle Ground: Reforming the Antidumping Laws in North America, Otawa, Centre for Trade Policy and Law, 1997.

Lindsey, B. y D. Ikeson, "Coming Home to Roost: Proliferating Antidumping Laws and the Growing Threat to U.S. Exports", Cato Trade Studies, núm. 14, 30 de julio, 2001.

Mastel, G., Antidumping Laws and the U.S. Economy, Washington D.C., Economic Strategy Institute, 1998.

Morke, M.F. y K.H. Kelley, Effects of Unfair Trade Imports on Domestic Industries: Us Antidumping and Countervailing Duty cases 1980-1988,
Washington, D.C., Federal Trade Commission, 1994.

Prusa, T.J., "On the Spread and Impact of Antidumping", Working Papers 7404, Cambridge, National Bureau of Economic Research, 1999.

_ , "Panorama general del impacto de las leyes estadounidenses de prácticas desleales de comercio internacional", B. Leycegui, W.B.P. Robson y S.D. Stein (editores), Comercio a golpes. Las prácticas desleales de comercio internacional bajo el TLCAN, México, ITAM-Miguel Porrúa, 1997.

Ragosta, J.A. y J.R. Magnus, "Antidumping and Antitrust Reform in the NAFTA: Beyond Rhetoric and Mischief", M. Hart (editor) Finding Middle Ground: Reforming the Antidumping Laws in North America, Otawa, Centre for Trade Policy and Law, 1997.

Russell, B.R., "How Long Can You Treat Water? The Anti-Economics of Trade Remedy Law", en M. Rodriguez, P. Low y B. Kotschwar (editores), Trade Rules in the Making. Challenges in Regional and Multilateral Negotiations, Washington D.C., Organization of the American States y Brookings Institution Press, 1999.

Schott, J.J., Prospects for Free Trade in the Americas, Washington D.C., Institute for International Economics, 2001.

SELA, Perspectivas de los países de América Latina y el Caribe en el ALCA, Secretaría Permanente del SELA (SP/DRE/D), núm. 27-98, agosto, 1998.

Stiglitz, J.E., "Dumping on Free Trade: The U.S. Import Trade Laws", Southern Economic Journal, vol. 64, núm. 2, pp. 402-424, 1997.

Tavares, J., C. Macario y K. Steinfatt, “Antidumping in the Americas", Serie Comercio Internacional núm. 12, CEPAL, Santiago, marzo, 2001.

uscBo, Antidumping Actions in the United States and Around the World: An Update, Washington D.C., United States Congressional Budget Office, junio, 2001.

Usitc, "The Economic Effects of Antidumping and Countervailing Duty Orders and Suspension Agreements", Investigation núm. 332-344, Washington D.C., United states International Trade Commission, Publication 2900, 2001.

USTR, Summary of the United States Negotiating Positions in the FTAA, Washington D.C., United States Trade Representative Office, 17 de enero, 2001.

Viner, J., Dumping: A Problem in International Trade, Chicago, University of Chicago Press (reimpreso por Augustus M. Kelly, Nueva York), 1923. 


\section{Anexo}

\section{Borrador de Tratado del ALCA}

\section{Capítulo sobre Subsidios, Antidumping y Derechos Compensatorios}

\section{(Sintesis)}

\section{Disposiciones generales}

Art. 1 [Las Partes solamente podrán iniciar procedimientos de investigación, [exámenes] y aplicar derechos antidumping [y compensatorios cuando corresponda], en perjuicio de mercancías de otra Parte, cuando la autoridad investigadora del país importador haya actuado de plena conformidad con las disposiciones estipuladas en el presente Capítulo. [Para todo lo no contemplado expresamente en el presente Capítulo], el Acuerdo relativo a la Aplicación del Artículo vi del Acuerdo General sobre Aranceles Aduaneros y Comercio de 1994 de la Organización Mundial del Comercio.

\section{Determinación de la existencia de dumping}

Art. 2 [Se considerará que un producto es objeto de dumping, es decir, que se introduce en el mercado de otro país a un precio inferior a su valor normal, cuando su precio de exportación al exportarse de un país a otro sea menor que el precio comparable, en el curso de operaciones comerciales normales, de un producto similar destinado al consumo en el país exportador.] [Los costos se calcularán normalmente sobre la base de los registros que lleve el exportador o productor objeto de investigación, siempre que tales registros estén en conformidad con los principios de contabilidad generalmente aceptados del país exportador y reflejen razonablemente los costos asociados a la producción y venta del producto considerado. Las autoridades tomarán en consideración todas las pruebas disponibles de que la imputación de los costos ha sido la adecuada, incluidas las que presente el exportador o productor en el curso de la investigación, siempre que esas imputaciones hayan sido utilizadas tradicionalmente por el exportador o productor, sobre todo en relación con el establecimiento de períodos de amortización y depreciación adecuados y deducciones por concepto de gastos de capital y otros costos de desarrollo. [Cuando no exista precio de exportación, o cuando, a juicio de la autoridad competente, el precio de exportación no 
sea fiable por existir una asociación o un arreglo compensatorio entre el exportador y el importador o un tercero, el precio de exportación podrá reconstruirse sobre la base del precio al que los productos importados se revendan por vez primera a un comprador independiente [únicamente si se demuestra que dicha relación afecta al precio de exportación] o, si los productos no se revendiesen a un comprador independiente o no lo fueran en el mismo estado en que se importaron, sobre una base razonable que la autoridad determine.]

\section{Determinación de la existencia de daño}

Art. 3 [La determinación de la existencia de daño se basará en pruebas positivas y comprenderá un examen objetivo: $a$ ) del volumen de las importaciones objeto de dumping y del efecto de éstas en los precios de productos similares en el mercado interno y $b$ ) de la consiguiente repercusión de esas importaciones sobre los productores nacionales de tales productos. [Para tales efectos no se permitirá ni se considerará como objetivo un examen que se fundamente en la utilización de estadísticas sobre grupos agregados de productos dentro del cual se encuentre el producto similar sujeto a investigación.]]

\section{Definición de rama de producción nacional}

Art. 4 [La expresión "rama de producción nacional" se entenderá en el sentido de abarcar a la totalidad de los productores domésticos del producto similar], o [ cuando ello no sea posible,] aquellos de entre ellos cuya producción conjunta constituya una proporción importante de la producción nacional total de dichos productos.]

\section{Iniciación y procedimiento de investigación}

Art. 5 [No se iniciará una investigación si las autoridades no han determinado, basándose en el examen del grado de apoyo o de oposición a la solicitud expresado por los productores nacionales del producto similar, que la solicitud ha sido hecha por o en nombre de la rama de producción nacional. La solicitud se considerará hecha "por la rama de producción nacional o en nombre de ella" cuando esté apoyada por productores nacionales cuya producción conjunta represente más del 50 por ciento de la producción total del producto similar.] [En el caso de productos exportados por pequeñas economías] [o cuando existan pruebas razonables que indiquen que el supuesto subsidio es una medida gubernamental de asistencia, directa o indirecta, para fomentar el desarrollo rural, mejorar la capacidad productiva o diversificar las inversiones en las economías más pequeñas del ALCA] no se considerará dumping. Cuando la autoridad determine que el margen de dumping es de minimis, o que el volumen de las importaciones reales o potenciales objeto de dumping o el daño son insignificantes, se pondrá inmediatamente fin a la investigación. [Para los efec- 
tos anteriormente expuestos] se considerará de minimis el margen de dumping cuando sea inferior al [5\%], expresado como porcentaje del precio de exportación. [Asimismo] se considerará insignificante el volumen de las importaciones objeto de dumping cuando se establezca que las procedentes de [de una pequeña economía] representan menos del [10\%] [7\%] de las importaciones del producto similar en la Parte importadora, salvo que los países que individualmente representan menos del [6\%] [7\%] de las importaciones del producto similar en la Parte importadora representen en conjunto más del [15\%] por ciento de esas importaciones.

\section{Pruebas}

Art. 6 [Durante toda la investigación antidumping, todas las partes interesadas tendrán plena oportunidad de defender sus intereses. A este fin, las autoridades darán a todas las partes interesadas, previa solicitud, la oportunidad de reunirse con aquellas partes que tengan intereses contrarios para que puedan exponerse tesis opuestas y argumentos refutatorios. [La fecha de celebración de la audiencia se notificará a las partes interesadas con por lo menos 15 días hábiles de anticipación. En la celebración de la audiencia se habrán de tener en cuenta la necesidad de salvaguardar el carácter confidencial de la información y la conveniencia de las partes. Ninguna parte estará obligada a asistir a una reunión, y su ausencia no irá en detrimento de su causa. Las partes interesadas tendrán también derecho, previa justificación, a presentar otras informaciones oralmente.] Al formular las determinaciones, la autoridad investigadora tendrá en cuenta toda la información verificable, presentada adecuadamente de modo que pueda utilizarse en la investigación sin dificultades excesivas, facilitada a tiempo y, cuando proceda, en el medio informático que haya solicitado la autoridad investigadora.

\section{Medidas provisionales}

Art. 7 [Sólo podrán aplicarse medidas provisionales si: i) se ha iniciado una investigación de conformidad con las disposiciones del artículo 5, se ha dado un aviso público a tal efecto y se han dado a las partes interesadas oportunidades adecuadas de presentar información y hacer observaciones; $i$ i) se ha llegado a una determinación preliminar positiva de la existencia de dumping y del consiguiente daño a una rama de producción nacional; y iii) la autoridad competente juzga que tales medidas son necesarias para impedir que se cause daño durante la investigación. [Las medidas provisionales se aplicarán por el período más breve posible, que no podrán exceder de cuatro meses, o, por decisión de la autoridad competente, a petición de exportadores que representen un porcentaje significativo del comercio de que se trate, por un período que no excederá de seis meses.] 


\section{Compromisos}

Art. 8 [Se podrán suspender o dar por terminados los procedimientos sin imposición de medidas provisionales o derechos antidumping si el exportador comunica que asume voluntariamente compromisos satisfactorios de revisar sus precios o de poner fin a las exportaciones a la zona en cuestión a precios de dumping, de modo que las autoridades queden convencidas de que se elimina el efecto perjudicial del dumping. Los aumentos de precios estipulados en los compromisos relativos a precios asumidos de forma voluntaria por los exportadores], no serán superiores a lo necesario para compensar el margen de [daño].

\section{Establecimiento y percepción de derechos}

Art. 9 [La decisión de establecer o no un derecho antidumping en los casos en que se han cumplido todos los requisitos para su establecimiento, y la decisión de fijar la cuantía del derecho antidumping en un nivel igual o inferior a la totalidad del margen de dumping, habrán de adoptarlas las autoridades de la Parte importadora. Es deseable que el establecimiento del derecho sea facultativo en el territorio de todas las Partes. Las autoridades deberán establecer un derecho antidumping [sea este provisional o definitivo] inferior al margen de dumping si ese derecho inferior basta para eliminar el daño a la rama de producción nacional.]

\section{Duración y examen de los derechos definitivos y de los compromisos}

Art. 11 [Cuando ello esté justificado, las autoridades examinarán la necesidad de mantener el derecho, por propia iniciativa o, siempre que haya transcurrido un período prudencial desde el establecimiento del derecho antidumping definitivo, a petición de cualquier parte interesada que presente informaciones positivas probatorias de la necesidad del examen. Las partes interesadas tendrán derecho a pedir a las autoridades que examinen si es necesario mantener el derecho para neutralizar el dumping, si sería probable que el daño siguiera produciéndose o volviera a producirse en caso de que el derecho fuera suprimido o modificado, o ambos aspectos. En caso de que, a consecuencia de un examen realizado de conformidad con el presente párrafo, las autoridades determinen que el margen de dumping es de minimis, o que el volumen de las importaciones, reales o potenciales, objeto de dumping o el daño es insignificante, conforme a la definición prevista en el párrafo 8 del artículo 5, el derecho antidumping] deberá suprimirse inmediatamente.]

\section{Países en desarrollo}

Art. 15 [Se reconoce que los países desarrollados Parte del Acuerdo del ALCA deberán tener particularmente en cuenta la especial situación de los países en desarrollo Parte del Acuerdo del ALCA cuando contemplen la aplicación de medidas antidumping [o compensatorias] en virtud del presente Acuerdo. Antes de la aplicación de derechos antidumping [o 
compensatorios] se [hará a los exportadores una propuesta de compromiso de precios o se dará oportunidad para que el país exportador elimine los programas de subvención, según sea el caso. La autoridad investigadora dará oportunidad adecuada para celebrar consultas en las que se discuta la propuesta de compromiso de precios o eliminación de los programas de subvención o cualquier otra solución constructiva permitida por este Acuerdo u otros tratados internacionales.]

\section{Eliminación de medidas antidumping}

Art. 19 [Cuando se establezca la zona de libre comercio y los bienes circulen en los países del ALCA fundamentalmente libres de restricciones, los países renunciarán al uso de medidas antidumping para el comercio recíproco.]

\section{De las medidas antidumping y compensatorias}

[Ninguna Disposición de otro Capítulo de este Tratado se interpretará en el sentido de imponer obligaciones a las Partes con respecto a sus disposiciones jurídicas sobre cuotas antidumping y compensatorias. Cada una de las Partes se reserva el derecho de aplicar sus disposiciones jurídicas en materia de cuotas antidumping y compensatorias a los bienes que se importen del territorio de cualquiera de las otras Partes.]

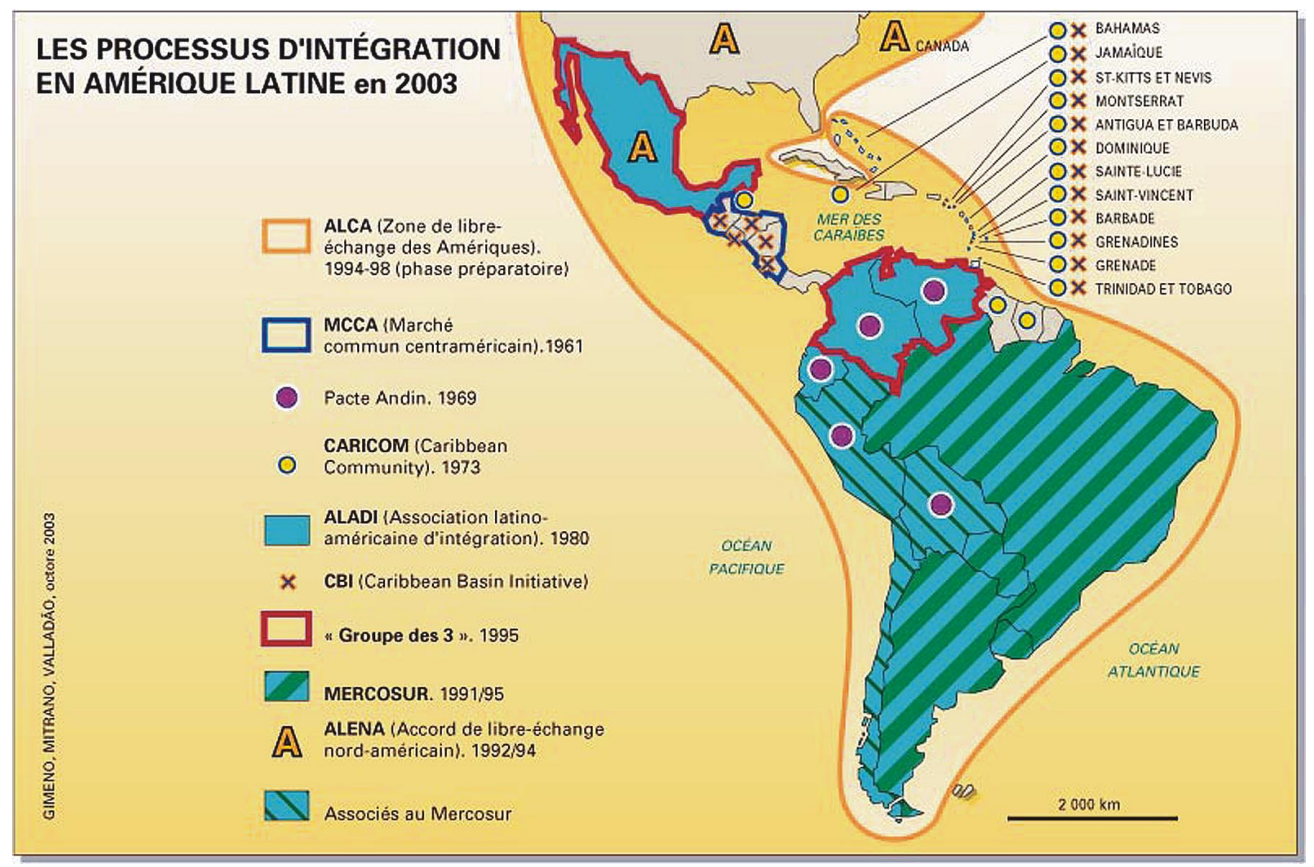

